\title{
O BRINCAR DURANTE O PERÍODO DE HOSPITALIZAÇÃO PARA TRATAMENTO DE CÂNCER PEDIÁTRICO
}

Recebido em: $16 / 11 / 2019$

Aprovado em: 26/06/2020 Licença:@) (1) @

Talita Grazielle Pires de Carvalho ${ }^{1}$ Universidade de Pernambuco (UPE)

Recife - PE - Brasil

Ameliane da Conceição Reubens-Leonidio ${ }^{2}$ Instituto Federal de Pernambuco (IFPE) - Campus Cabo de Santo Agostinho Cabo de Santo Agostinho - PE - Brasil

Priscilla Pinto Costa da Silva Universidade Federal do Rio Grande do Norte (UFRN)

Natal - RN - Brasil

Clara Maria Silvestre Monteiro de Freitas ${ }^{4}$

Universidade de Pernambuco (UPE)

Recife - PE - Brasil

Pierre Normando Gomes-da-Silva ${ }^{5}$ Universidade Federal da Paraíba (UFPB)

João Pessoa - PB - Brasil

Ana Raquel Mendes dos Santos 6

Universidade de Pernambuco (UPE)

Recife - PE - Brasil

\footnotetext{
${ }^{1}$ Mestre e Doutoranda em Educação Física pelo Programa Associado de Pós-graduação em Educação Física Universidade de Pernambuco/Universidade Federal da Paraíba. Grupo de Estudos e Pesquisas Socioculturais: o indivíduo em educação física e desporto.

${ }^{2}$ Mestre e Doutoranda em Educação Física pelo Programa Associado de Pós-graduação em Educação Física Universidade de Pernambuco/Universidade Federal da Paraíba. Grupo de Estudos e Pesquisas Socioculturais: o indivíduo em educação física e desporto. Professora EBTT - IFPE campus Cabo de Santo Agostinho.

${ }^{3}$ Doutora em Educação Física. Professora titular da Universidade Federal do Rio Grande do Norte.

4 Doutora e Pós Doutora em Educação Física pela Universidade do Porto. Professora adjunta da Universidade de Pernambuco. Professora associada do Programa Associado de Pós-Graduação em Educação Física UPE/UFPB. Líder do Grupo de Estudos e Pesquisas Socioculturais.

${ }^{5}$ Doutor em Educação e Pós Doutor em Educação Física. Professor Titular da Universidade Federal da Paraíba e do Programa Associado de Pós-Graduação em Educação Física UPE/UFPB. Líder do Grupo de Pesquisas em Pedagogia da Corporeidade (GEPEC/CNPq, desde 2006) e do Laboratório de ensinopesquisa-extensão ESCOLA BRINCANTE.

6 Doutora em Educação Física. Professora assistente da Universidade de Pernambuco. Docente colaboradora do Programa Associado de Pós-Graduação em Educação Física UPE/UFPB. Grupo de Estudos e Pesquisas Socioculturais: o indivíduo em educação física e desporto
} 
RESUMO: Buscou-se analisar a percepção de crianças e adolescentes sobre o brincar durante a hospitalização para tratamento de câncer. Esse estudo descritivo de abordagem qualitativa, envolveu 14 pacientes com idade entre 7 e 17 anos, internados em um hospital de Recife, Pernambuco. $\mathrm{Na}$ coleta dos dados, utilizou-se um questionário sociodemográfico e um protocolo de consulta ao prontuário para caracterizar os sujeitos, a entrevista semiestruturada e o diário de campo para analisar as percepções a partir de Bardin. Os resultados apontaram que o brincar faz parte do universo da hospitalização podendo ser um recurso humanizado para o enfrentamento desta realidade. Além disso, notou-se a necessidade de ampliação do brincar contemplando também movimentos corporais e identificou-se uma possibilidade de resposta a essa necessidade através da utilização do videogame ativo.

PALAVRAS-CHAVE: Jogos e Brinquedos. Câncer. Hospitalização. Humanização.

\section{PLAYING DURING THE PERIOD OF HOSPITALIZATION FOR TREATMENT OF PEDIATRIC CANCER}

ABSTRACT: Searched to analyze the perception of children and adolescents about playing during hospitalization for cancer treatment. This descriptive study with a qualitative approach, involved 14 patients aged between 7 and 17 years, admitted to a hospital in Recife, Pernambuco. In the data collection, a sociodemographic questionnaire and a protocol for consulting the medical records were used to characterize the subjects, the semi-structured interview and the field diary to analyze the perceptions from Bardin. The results showed that playing is part of the universe of hospitalization and can be a humanized resource to face this reality. In addition, there was a need to expand playing, also including body movements, and a possibility of responding to this need was identified through the use of the active video game.

KEYWORDS: Play and Playthings. Cancer. Hospitalization. Humanization.

\section{Introdução}

Ao se deparar com o diagnóstico de câncer, crianças e adolescentes vivenciam diversas situações de sofrimento. O contexto da doença, do tratamento e a possível perspectiva de morte, repercutem de maneira impactante na vida destes pacientes trazendo significativas perdas nos âmbitos: 1 . social, com o distanciamento da família e amigos; 2. emocional, com a baixa autoestima; e 3. físico, com náuseas, vômitos, reações alérgicas, alterações na pele, fadiga, perda de apetite, dor, perda de pelos e cabelos, além do aumento do risco de infecções, hemorragias e complicações tromboembólicas que podem ser sentidas durante ou logo após o tratamento (MITRE; GOMES, 2004; MELO; 
O Brincar Durante o Período de Hospitalização para Tratamento de Câncer Pediátrico Talita Grazielle P. de Carvalho, Ameliane da C. Reubens-Leonidio, Priscilla Pinto C. da Silva, Clara Maria S. M. de Freitas, Pierre N. Gomes-da-Silva e Ana Raquel M. dos Santos

VALLE, 2010; NEHMY et al. 2011; MALTA; SCHALL, 2012; CATAUDELLA; ZELCER，2012；DUARTE；ZANINI; NEDEL，2012；ALVES；GUIRARDELLO; KURASHIMA, 2013).

Concomitantemente, há uma redução na frequência das atividades cotidianas, dentre elas as atividades lúdicas, como o brincar. Winnicott (1975) destaca a brincadeira como universal e própria da saúde, visto que o brincar facilita o crescimento e conduz a relacionamentos grupais, constituindo uma forma de comunicação consigo e com os outros. Apresenta-se como uma forma de presença, de habitar o espaço/tempo onde a criança se mantém imersa, atenta, perceptiva e ativa na circunstância da brincadeira. Segundo Gomes-da-Silva (2016, p. 157), “a conduta lúdica nem é do controle, nem da submissão, mas da unidade experimentada na absorção do jogar" e nesse sentido o jogo é tido como privilegiado por sua capacidade de absorver o jogador para dentro da sua realidade, de maneira vivaz, saudável e potente para criar um relacionamento com a realidade interna e externa. Ao interagir com o mundo real, com o mundo da brincadeira e com os outros, a criança externaliza suas proposições e fabulações, transformando o mundo a seu modo (GOMES-DA-SILVA, 2011).

Assim, o brincar deve ser considerado não apenas no que se refere à ordem curativa e à redução do tempo de permanência no hospital, mas também no sentido de apoiar a criança e o adolescente para atravessar a situação de doença e hospitalização, através de vivências prazerosas e saudáveis. Há, portanto, uma necessidade de humanização das práticas de saúde no ambiente hospitalar, o que impulsionou no Sistema Único de Saúde (SUS), a formulação do Programa Nacional de Humanização da Assistência Hospitalar - PNHAH (2002) e da Política Nacional de Humanização da Atenção e Gestão, compondo a rede HumanizaSUS (BRASIL, 2006), além da sanção da Lei nº 11.104/2005, também chamada Lei da Brinquedoteca, para criação de um "espaço 
O Brincar Durante o Período de Hospitalização para Tratamento de Câncer Pediátrico Talita Grazielle P. de Carvalho, Ameliane da C. Reubens-Leonidio, Priscilla Pinto C. da Silva, Clara Maria S. M. de Freitas, Pierre N. Gomes-da-Silva e Ana Raquel M. dos Santos

provido de brinquedos e jogos educativos, destinado a estimular as crianças e seus acompanhantes a brincar”, durante a hospitalização (BRASIL, 2005).

Uma ação mais humanizada no ambiente hospitalar pode amenizar dor e sofrimento independente da faixa etária do paciente (ISAYAMA et al., 2011). A criança neste contexto não deixa de ser criança (PAGOTTO; SILVA, 2006), sendo o brincar um importante recurso para o seu desenvolvimento e humanização no período de hospitalização, utilizado como objeto de vários estudos como os de Lima, et al. (2008), Isayama et al. (2011) e Carvalho e Freitas (2018) que apresentam ações com base no brincar, desenvolvidas por profissionais de educação física no âmbito hospitalar em nível de ambulatório e enfermaria pediátrica, com o intuito de auxiliar na humanização do atendimento.

Ao introduzir possibilidades para o brincar, o ambiente hospitalar permite a construção de novos significados para a situação vivida, superando a centralidade na doença. Desse modo, buscou-se analisar a percepção de crianças e adolescentes sobre o brincar durante a hospitalização para tratamento de câncer.

\section{Material e Métodos}

Trata-se de um estudo descritivo de abordagem qualitativa, tendo como cenário a brinquedoteca do Centro Hospitalar de Onco-Hematologia Pediátrica (CEONHPE) do Hospital Universitário Oswaldo Cruz (HUOC), localizado no Município do Recife Pernambuco, sendo um recorte da pesquisa intitulada "Videogame ativo como estratégia no enfrentamento do câncer infantojuvenil: uma perspectiva da educação física", que ocorreu entre maio e junho de 2015, contando com a participação de 14 pacientes. 
O Brincar Durante o Período de Hospitalização para Tratamento de Câncer Pediátrico Talita Grazielle P. de Carvalho, Ameliane da C. Reubens-Leonidio, Priscilla Pinto C. da Silva, Clara Maria S. M. de Freitas, Pierre N. Gomes-da-Silva e Ana Raquel M. dos Santos

A caracterização dos sujeitos se deu a partir da aplicação de um questionário sociodemográfico, respondido por um adulto responsável, e da utilização de um protocolo de consulta ao prontuário do paciente, gerando dados categóricos.

Para uma melhor compreensão do fenômeno estudado e maior qualidade na pesquisa foi realizada a triangulação dos dados qualitativos de dois instrumentos de coleta (FLICK, 2009): uma entrevista semiestruturada com o paciente e o diário de campo.

A entrevista semiestruturada com o paciente foi utilizada por ser uma técnica eficiente para obtenção de dados em profundidade, que pode ser aplicada entre pessoas que não sabem ler e escrever, permitindo identificar dúvidas e estabelecer uma relação de confiança (OLIVEIRA et al., 2010). Os dados foram registrados por meio de um aparelho gravador de voz digital, que permitiu o arquivamento e posteriormente a transcrição. Foram adotados os critérios sugeridos por Flick (2009): 1. realizou-se uma breve introdução sobre o procedimento e o tema abordado; 2. as perguntas utilizadas foram acessíveis aos participantes do estudo e 3. cuidou-se para não influenciar os entrevistados com opiniões particulares ou tendenciosas.

O uso do diário de campo buscou complementar as informações das entrevistas, através de um registro minucioso do dia a dia da pesquisa, sendo preenchido pelo pesquisador responsável. O recurso foi utilizado durante as observações realizadas na brinquedoteca, permitindo identificar situações envolvendo o brincar, que por vezes foram captadas de maneira superficial na entrevista. Possibilitou-se assim uma maior reflexão acerca do objeto de estudo, a fim de rever seus limites e desafios, auxiliando no aprofundamento do conhecimento do fenômeno estudado. No diário de campo, foram registradas as situações vivenciadas e interações ocorridas entre pacientes, profissionais de saúde, brinquedistas e cuidadores/familiares. 
O Brincar Durante o Período de Hospitalização para Tratamento de Câncer Pediátrico Talita Grazielle P. de Carvalho, Ameliane da C. Reubens-Leonidio, Priscilla Pinto C. da Silva, Clara Maria S. M. de Freitas, Pierre N. Gomes-da-Silva e Ana Raquel M. dos Santos

Na análise do conteúdo das entrevistas e do diário de campo, utilizou-se Bardin (2009), por meio dos seguintes passos: 1. Construção do corpus de análise a partir da transcrição do material coletado nas entrevistas e do material registrado no diário de campo; 2. "leitura flutuante" para familiarização com os conteúdos do material coletado; 3. definição e identificação das unidades de registros no texto; 4. nomeação e identificação das unidades de significação; 5. quantificação das unidades de significação em cada corpus; 6. agrupamento dos temas para construção das categorias; 7. nomeação, quantificação e descrição das categorias; e 8. associação das categorias às características dos entrevistados e ao conhecimento científico. Nos resultados, a frequência das unidades de registro, oriundas das falas dos participantes do estudo, será apresentada entre parênteses, logo após a descrição da atividade.

Foram analisadas 3 categorias instituídas a priori: 1. principais atividades realizadas durante a hospitalização; 2 . brincadeiras preferidas durante a hospitalização e 3. atividades que os pacientes gostariam de realizar durante o período de hospitalização. Para a apresentação gráfica dos resultados foi utilizado o software GoDiagram Express versão 2.6.2.

Com o intuito de diferenciar as falas, os pacientes foram identificados por meio da letra "P", seguida pelo sexo representado pelas letras "F" para feminino e "M" para masculino, pelo número e idade do paciente no dia da entrevista.

Esse estudo foi aprovado pelo Comitê de Ética em Pesquisa do HUOC, com número do CAAE 42625315.5.0000.5207 e parecer 1.013.574. A fim de respeitar as questões éticas da Resolução 466/12 do Conselho Nacional de Saúde/MS, que regulamenta as diretrizes e normas de pesquisa envolvendo seres humanos. Os sujeitos que aceitaram participar como voluntários tiveram o Termo de Consentimento Livre e 
O Brincar Durante o Período de Hospitalização para Tratamento de Câncer Pediátrico Talita Grazielle P. de Carvalho, Ameliane da C. Reubens-Leonidio, Priscilla Pinto C. da Silva, Clara Maria S. M. de Freitas, Pierre N. Gomes-da-Silva e Ana Raquel M. dos Santos

Esclarecido e o Termo de Assentimento devidamente assinados por seus responsáveis legais por serem menores de 18 anos.

\section{Resultados e Discussão}

A amostra do estudo foi composta por 14 pacientes, com idade entre 7 e 17 anos. Destes, 10 eram do sexo masculino e 4 do sexo feminino. Do total dos sujeitos participantes do estudo 7 foram diagnosticados com Leucemia Linfóide Aguda, 4 com Osteossarcoma, 1 com Leucemia Mielóide Aguda, 1 com Linfomas de Grandes Células e 1 com Neuroblastoma. Dentre os pacientes, 2 apresentaram amputação em um dos membros inferiores. Todos os pacientes estavam internados no hospital há mais de cinco dias, tempo considerado suficiente para que possíveis prejuízos psicológicos pudessem se instalar, trazendo riscos ao desenvolvimento (DIAS; BAPTISTA; BAPTISTA, 2003).

No tocante à renda familiar, 8 dos responsáveis pelos pacientes declararam ter uma renda familiar mensal de até 1 salário mínimo, enquanto 6 declararam possuir renda familiar de até 4,5 salários mínimos. Em relação ao local de residência, 9 dos pacientes residiam no Estado de Pernambuco, 2 em Alagoas, 1 na Bahia, 1 na Paraíba e 1 em Sergipe, dos quais 10 pacientes residiam em cidades do interior destes estados.

\section{Principais Atividades Realizadas Durante a Hospitalização e o Brincar como Estratégia de Enfrentamento}

Em relação aos tipos de atividades que os pacientes mais realizavam, foi criada a categoria "Principais atividades realizadas durante o período de hospitalização", representada pelo balão na cor cinza escuro. Desta categoria, emergiram duas subcategorias, representadas pelos balões cinza claro: 1. Atividades da vida diária mais realizadas; e 2. Atividades de lazer mais realizadas, destacadas na Figura 1. 


\section{Figura 1: Principais atividades realizadas durante o período de hospitalização}

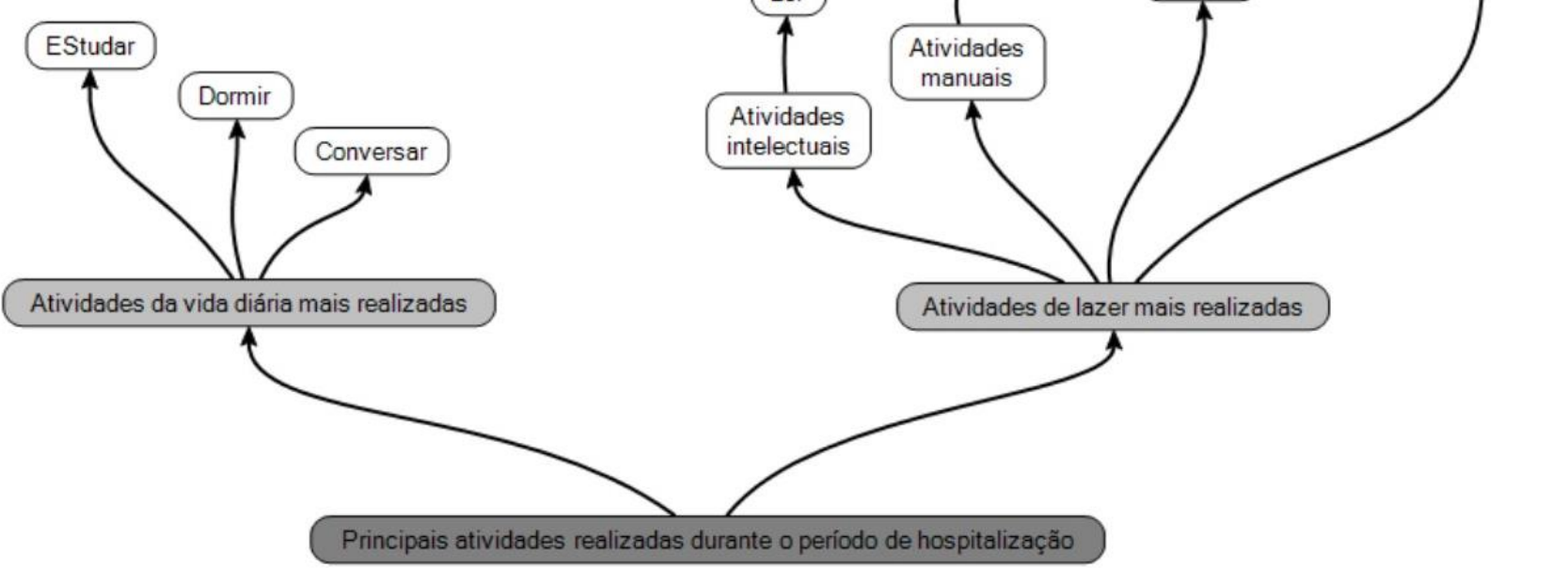

Fonte: Dados da pesquisa

Ao serem questionados "o que você tem feito, durante o tempo em que você fica no hospital?", os pacientes relataram:

[...] eu passo a maior parte do tempo deitada! Só mexendo no celular ou assistindo televisão... Ah! Tem a sala de aula! (P.F.7-14 anos).

[...] converso, durmo e assisto e também uso muito meu celular (P.M.2-14 anos).

[...] eu durmo, assisto, agora tô jogando no meu notebook (P.M.11-11 anos).

[...] há quatro meses no hospital... Eu fico só no videogame, brincando, desço pra brinquedoteca, brinco na brinquedoteca, fico desenhando só se 'intertendo' (entretendo). Eu jogo videogame no Xbox (joystick), jogo no celular, assisto, desenho, leio e só! (P.M.6 de 16 anos).

Nota-se, portanto, que as atividades da vida diária mais realizadas são estudar (08), dormir (04) e conversar (03). O direito ao atendimento educacional especial está ancorado pela Lei 13.716, de 2018 que assegura aos alunos da educação básica, que estejam internados por tempo prolongado para tratamento de saúde, o atendimento educacional que lhes é garantido pela Lei de Diretrizes e Bases da Educação Brasileira 
O Brincar Durante o Período de Hospitalização para Tratamento de Câncer Pediátrico Talita Grazielle P. de Carvalho, Ameliane da C. Reubens-Leonidio, Priscilla Pinto C. da Silva, Clara Maria S. M. de Freitas, Pierre N. Gomes-da-Silva e Ana Raquel M. dos Santos

(Lei n9.394, de 20 de dezembro de 1996). Por outro lado, os estudos de Winter et al. (2009) e Götte et al. (2014) apontaram que durante o período de hospitalização para tratamento de câncer, as crianças têm uma redução significativa das atividades que incluem movimentações corporais comuns no dia a dia. Durante esse período mais de $50 \%$ dos pacientes chegam a passar cerca de 23 horas por dia no leito (GÖTTE et al., 2014). É nesse contexto que dormir e conversar passam a se configurar, também, como atividades da vida diária mais realizadas.

Dentre as atividades de lazer mais realizadas, assistir - TV, tablet e celular (12), atividades manuais - pintar/desenhar (03), atividades intelectuais - ler (02) e atividades lúdicas - brincar (11) foram relatadas, corroborando os estudos de Motta e Enumo (2004), Hostert; Enumo e Motta Loss (2014) e Mota Pacciulio Sposito et al. (2018), que apontam o brincar com brinquedo, jogos de regras, ler, pintar, desenhar e assistir televisão como as atividades mais realizadas por pacientes entre 6 e 12 anos durante o tratamento de câncer.

O brincar, para os pacientes do CEONHPE que participaram da pesquisa, aparece de diversas formas, através de jogos, na brincadeira de casinha, de boneca, de carro, massa de modelar ou bola de gude, mostrando que as crianças brincam no hospital e são criativas para inserir a brincadeira no contexto da hospitalização, adaptando-se às limitações do ambiente, ao tempo de internação e às dificuldades físicas e emocionais decorrentes da doença e do tratamento.

Assim, o brincar revela-se extremamente importante nesse contexto, pois permite um espaço onde a criança pode resistir a tentativa de mutilação social dos sentidos ocasionada pela vivência no ambiente hospitalar. Ao brincar, a criança atribui novas significações negando as rédeas e as prisões adultas que lhes foram reservadas e se renovam, liberando seus sentidos (LARIZZATTI, 2010). 
O Brincar Durante o Período de Hospitalização para Tratamento de Câncer Pediátrico Talita Grazielle P. de Carvalho, Ameliane da C. Reubens-Leonidio, Priscilla Pinto C. da Silva, Clara Maria S. M. de Freitas, Pierre N. Gomes-da-Silva e Ana Raquel M. dos Santos

Segundo Hostert; Motta e Enumo (2015), atividades como brincar, conversar, assistir televisão e estudar aparecem como alguns dos principais comportamentos de enfrentamento da hospitalização de pacientes com câncer com idade entre 6 e 12 anos. Tais atividades estão relacionadas a instâncias de coping entendidas como favoráveis ao processo de adaptação ao contex to hospitalar.

O coping, também chamado de estratégias ou processos de enfrentamento é tido para Ramos; Enumo e Paula apud Lazarus; Folkman7 (1984, p. 141) como:

[...] mudanças constantes nos esforços, cognitivos e comportamentais, utilizado pelos indivíduos com objetivo de lidar com demandas específicas, internas ou externas, que são avaliadas como sobrecarregando ou excedendo seus recursos pessoais.

Dessa forma, o coping é visto como um tipo de processo adaptativo que faz a mediação da relação entre a pessoa e o ambiente estressante. Nesta lógica, constituem-se as diferenças individuais e grupais na vulnerabilidade a eventos estressores, explicadas a partir da avaliação cognitiva (GALVÃO, 2014; RAMOS; ENUMO; PAULA, 2015).

Nesse sentido, o brincar pode se configurar como uma estratégia de enfrentamento da situação de doença/tratamento, modificando percepções sobre o ambiente hospitalar, tornando-o favorável ao desenvolvimento emocional do paciente brincante, e surgindo como uma estratégia para ressignificar o tempo, o espaço e a situação vivida.

\section{Brincadeiras Preferidas Durante a Hospitalização e as Limitações do Brincar Considerando o Movimento Corporal}

$\mathrm{Na}$ segunda categoria serão apresentadas as brincadeiras preferidas pelos pacientes durante o período de hospitalização, como mostra a Figura 2:

7 LAZARUS, R. S.; FOLKMAN, S. Stress, appraisal and coping. New York: Springer Publishing Company, 1984. 
O Brincar Durante o Período de Hospitalização para Tratamento de Câncer Pediátrico

Talita Grazielle P. de Carvalho, Ameliane da C. Reubens-Leonidio, Priscilla Pinto C. da Silva, Clara

Maria S. M. de Freitas, Pierre N. Gomes-da-Silva e Ana Raquel M. dos Santos

\section{Figura 2: Brincadeiras preferidas por crianças e adolescentes durante a hospitalização}

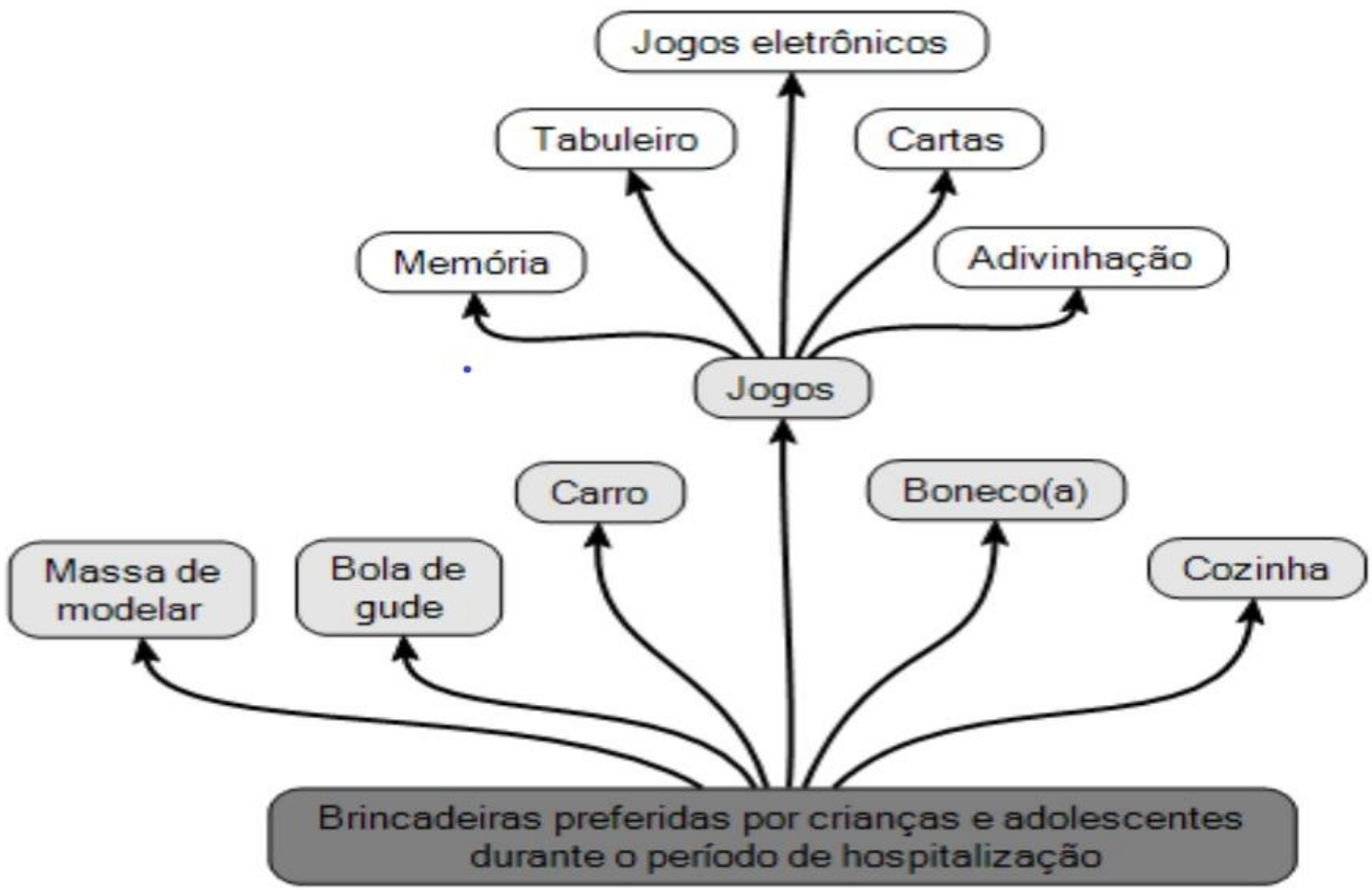

Fonte: Dados da pesquisa

Dentre as brincadeiras preferidas do grupo destacaram-se o brincar através de jogos - eletrônicos, tabuleiro, cartas, adivinhação, memória (11) e o brincar envolvendo atividades manuais (11) - brincar de carro, boneco (a), cozinha, massa de modelar e bola de gude, como mostram os relatos dos pacientes diante da pergunta: "Quais as atividades, brincadeiras ou jogos que você mais gosta de realizar durante o tempo que fica no hospital?"

[...] No hospital só o videogame mesmo! Também dominó e baralho, dama. O meu videogame, dominó... Só! (P.M.1-13 anos).

[...] Eu joguei hoje com meu colega o cara-a-cara, tem vários aqui. Eu gosto de jogar dominó, baralho (P.M.5-12 anos).

[...] Eu gosto mais de brincar de massinha de modelar. Eu brinco de boneco, de computador. Eu brinco com meus amigos e meus irmãos. Eu gosto de brincar do jogo dos carros, que a pessoa lava aí depois enxuga eles (P.M.10-7 anos). 
O Brincar Durante o Período de Hospitalização para Tratamento de Câncer Pediátrico Talita Grazielle P. de Carvalho, Ameliane da C. Reubens-Leonidio, Priscilla Pinto C. da Silva, Clara Maria S. M. de Freitas, Pierre N. Gomes-da-Silva e Ana Raquel M. dos Santos

É notável a preferência por jogos e brincadeiras nos quais há uma limitação do movimento corporal, seja por medo, superproteção dos pais, dificuldades por causa do acesso/cateter ou limitações físicas pelo tratamento ou pela amputação de algum membro corporal. Têm-se como exemplos os relatos do P.M.11 de 11 anos, que teve um dos membros inferiores amputado e do P.M.3 de 7 anos, que estava com um dos braços imobilizado:

\footnotetext{
[...] eu gostaria de brincar, me divertir mais. Tem uma, mas eu não posso. Jogar bola, por causa da perna! (P.M.11 de 11 anos).

[...] "Uno... bolinha de gude que eu consigo jogar com uma mão!(P.M.3 de 7 anos).
}

O hospital muitas vezes é visto por pacientes como um lugar de repouso e silêncio (HOSTERT; ENUMO; MOTTA LOSS, 2014). Um local no qual o brincar está restrito, onde as condições do ambiente, as dificuldades oriundas da doença e do tratamento limitam as possibilidades para exploração de movimentos corporais em jogos e brincadeiras.

Essa limitação do movimento corporal pode acarretar em consequências como fraqueza e atrofia do músculo esquelético, conjuntas contraturas, doença tromboembólica, inflamação sistêmica (BROWER, 2009) e um declínio na função física (STENHOLM et al., 2011), além de atitudes de regressão, estado depressivo e transtornos de comportamento oriundos da hospitalização (AJURIAGUERRA, 2005).

É importante destacar que na manifestação do brincar no ambiente hospitalar há uma valorização da expressão de pensamento e de sentimentos, em detrimento do movimento. Esses três pilares são para Medina et al. (2010) os elementos fundamentais para a legítima expressão do ser. Para Galvão (2014), o movimento corporal é um fator importante no desenvolvimento infantil, sendo visto como instrumento de exploração do meio físico, voltado à ação da criança para a realidade objetiva. 
O Brincar Durante o Período de Hospitalização para Tratamento de Câncer Pediátrico Talita Grazielle P. de Carvalho, Ameliane da C. Reubens-Leonidio, Priscilla Pinto C. da Silva, Clara Maria S. M. de Freitas, Pierre N. Gomes-da-Silva e Ana Raquel M. dos Santos

O movimento corporal também estabelece uma relação de reciprocidade com as emoções, uma vez que estas podem estar vinculadas à maneira como o tônus muscular se forma se conserva ou se consome, onde "a modelagem do corpo realizada pela atividade do tônus muscular permite além da exteriorização dos estados emocionais, a tomada de consciência dos mesmos pelo sujeito" (GALVÃO, 2014 p. 62 - 63).

Além das limitações apresentadas pelos pacientes, nem sempre o ambiente hospitalar é considerado adequado para brincadeiras que envolvam movimentos corporais. Há casos de pacientes que ficam restritos aos leitos e são impedidos pelos profissionais de saúde por não ter um espaço considerado como adequado no hospital para prática de brincadeiras como correr, dançar ou jogar bola. Neste sentido, faz-se necessário que os hospitais ofereçam espaços adaptados, materiais e profissionais capacitados para que crianças e adolescentes em tratamento sejam incentivadas e possam usufruir de uma ampla oferta de brincadeiras e jogos, incluindo uma variedade de movimentações corporais, que respeitem suas limitações.

No CEONHPE observou-se um ambiente humanizado com 2 brinquedotecas que atendiam os pacientes da enfermaria, sendo que uma delas funcionava como uma sala de aula da classe hospitalar SEMEAR. A brinquedoteca contava com um acervo de materiais lúdicos diversos, com oferta de livros, jogos e brinquedos, além de um videogame que ficava disponível para os pacientes jogarem acompanhados pelas brinquedistas do Grupo de Apoio à Criança Carente com Câncer (GAC-PE) responsáveis pelas atividades do local. Notou-se, contudo, que as atividades ofertadas durante o período da pesquisa, embora bastante importante para o desenvolvimento infantil, pouco exploravam o movimento corporal.

Para Paula (2013), apesar do aumento na oferta de atividades lúdicas nos hospitais, são muitas as limitações impostas às crianças e adolescentes relacionadas aos jogos e 
O Brincar Durante o Período de Hospitalização para Tratamento de Câncer Pediátrico Talita Grazielle P. de Carvalho, Ameliane da C. Reubens-Leonidio, Priscilla Pinto C. da Silva, Clara Maria S. M. de Freitas, Pierre N. Gomes-da-Silva e Ana Raquel M. dos Santos

brincadeiras com movimentos corporais, fato que aponta para a necessidade de discussões a respeito de uma abordagem diferenciada, como por exemplo, a partir da perspectiva da Educação Física nesse contexto.

Atividades que os Pacientes Gostariam de Realizar Durante o Período de Hospitalização e a Ampliação do Brincar Considerando o Movimento Corporal a

\section{Partir do Videogame Ativo}

$\mathrm{Na}$ terceira categoria "atividades que os pacientes gostariam de realizar durante o período de hospitalização", surgiram duas subcategorias: "atividades sem possibilidade de realização no ambiente hospitalar" e "atividades com possibilidade de realização no ambiente hospitalar", como aponta a Figura 3.

Figura 3: Atividades que os pacientes gostariam de realizar durante o período de hospitalização

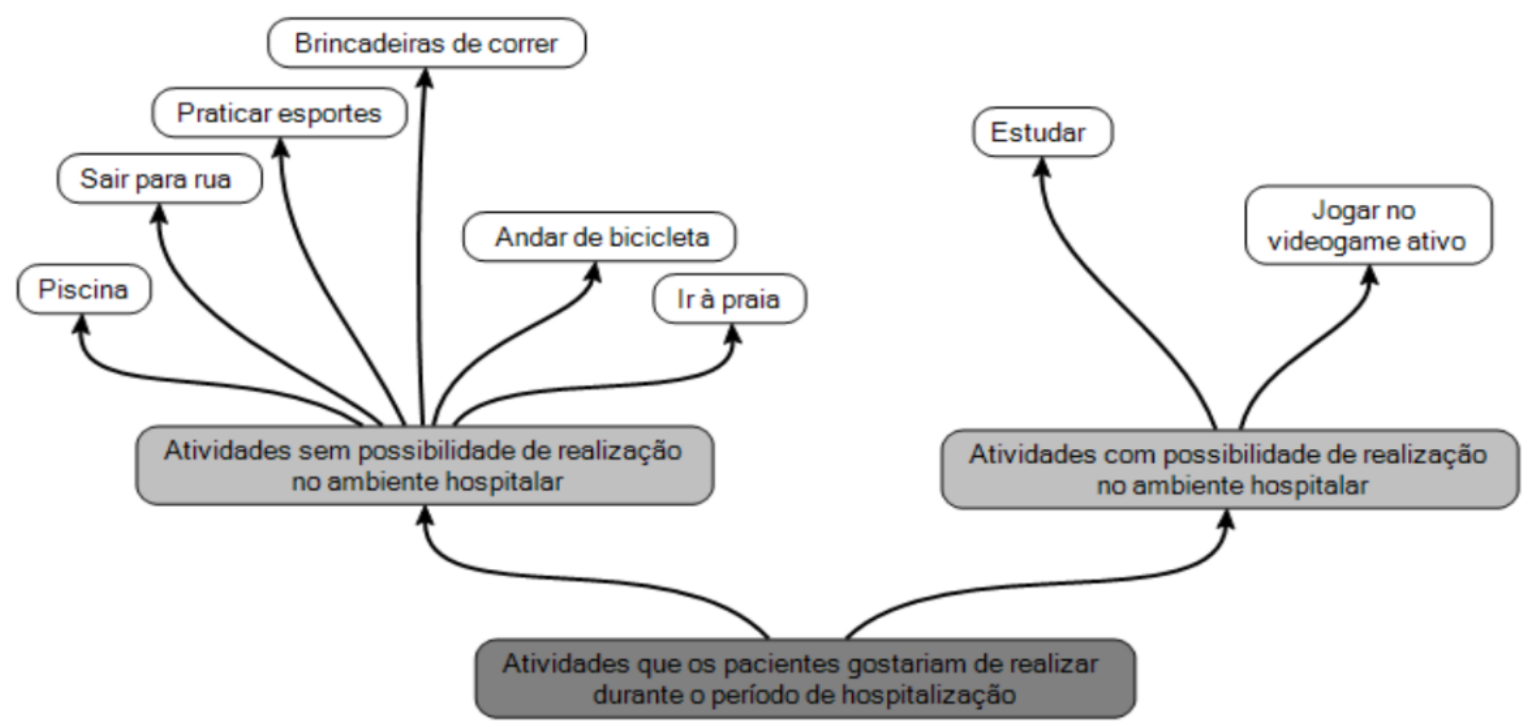

Fonte: Dados da pesquisa

Diante do questionamento "o que você gostaria de fazer durante o tempo em que fica no hospital?" foram citadas como atividades sem possibilidade de realização no ambiente hospitalar: brincadeiras de correr (07), praticar esportes (05), sair para rua (04), 
O Brincar Durante o Período de Hospitalização para Tratamento de Câncer Pediátrico Talita Grazielle P. de Carvalho, Ameliane da C. Reubens-Leonidio, Priscilla Pinto C. da Silva, Clara Maria S. M. de Freitas, Pierre N. Gomes-da-Silva e Ana Raquel M. dos Santos

andar de bicicleta (02), ir à praia (01) e à piscina (01), como aparecem nas citações a seguir:

[...] Dar uma volta, uma voltinha lá fora se pudesse (P.M.6 de 16 anos).

[...] eu gosto de correr, andar de bicicleta, fazer jiu-jitsu... Só tô esperando voltar a andar! (P.M.11 de 11 anos).

[...] ficar na piscina (risos) ir pra praia [...] (P.M.3 de 7 anos).

Os relatos de atividades sem possibilidade de realização no ambiente hospitalar apontam para a necessidade de ocupação de espaços mais amplos, com mais interação social e ambiental, além do movimento corporal, considerados importantes para o desenvolvimento físico e psicológico de crianças e adolescentes (GALVÃO, 2014; PAULA, 2013). Essas atividades são rotineiras para crianças e adolescentes, no entanto encontram-se limitadas pelo ambiente hospitalar, configurando mais como um desejo do que uma possibilidade de vivência real.

Para as atividades com possibilidade de realização no ambiente hospitalar, os participantes do estudo destacaram com mais frequência, estudar (05) e jogar no videogame ativo (03), como no seguinte depoimento:

[...] ir pra escola, porque eu vou pra escola todo dia quando eu 'tiver' em casa!(P.M.3 de 7 anos).

[...] com certeza seria praticar esporte, mas não dá pra fazer, mas jogar no Xbox também é legal! Ajuda muito a distrair (P.M.2 de 14 anos).

Com relação ao estudar, nota-se um desejo de estar na escola e interagir com outras pessoas, bem como realizar atividades educativas. Embora houvesse uma impossibilidade de interação com os amigos da escola, esse último desejo, no sentido da aprendizagem, pode ser suprido no acompanhamento pedagógico personalizado oferecido no hospital, através da classe hospitalar.

Sobre o jogar videogame ativo, este surge como uma necessidade de ampliar as possibilidades do brincar no ambiente hospitalar. Para Winnicott (1975), quando um 
O Brincar Durante o Período de Hospitalização para Tratamento de Câncer Pediátrico Talita Grazielle P. de Carvalho, Ameliane da C. Reubens-Leonidio, Priscilla Pinto C. da Silva, Clara Maria S. M. de Freitas, Pierre N. Gomes-da-Silva e Ana Raquel M. dos Santos

paciente encontra-se impossibilitado de brincar, se faz necessário atender a esse sintoma principal, tornando-o apto a brincar, para a partir da brincadeira interpretar fragmentos de sua conduta, uma vez que a necessidade do brincar é inerente à criança, sendo considerado como sinal de saúde, a qual está relacionada à sua saúde emocional.

O brincar configura-se, portanto, como uma experiência criativa a consumir espaço e tempo, intensamente real para quem brinca. Nestas ações, a criança manipula fenômenos externos a serviço do sonho e veste fenômenos externos escolhidos com significado e sentimento oníricos (WINNICOTT, 1975).

Carvalho e Freitas (2018), ao trazer para o hospital o olhar da Educação Física, por meio dos jogos de videogame ativo, puderam observar uma boa aceitação por parte dos pacientes, sendo estes jogos vistos como uma estratégia lúdica que contribui para despertar sentimentos como alegria e felicidade dentro de um ambiente permeado de doença, sofrimento e medo da morte (CARVALHO et al., 2018).

A partir dos relatos dos participantes do presente estudo e tomando como conceito de saúde a possibilidade de traçar um caminho pessoal e original, em direção ao bemestar físico, psíquico e social, não a vendo como um estado de estabilidade, sendo ela algo mutável todo o tempo (DEJOURS, 1986). Estratégias como o uso do videogame ativo para estimular movimentos, o brincar como um instrumento corporal e a vivência de práticas realizadas fora do ambiente hospitalar através do ambiente virtual contribui para ampliação da humanização e o enfrentamento da situação de hospitalização na busca da saúde.

\section{Considerações Finais}

Diante dos achados, foi possível observar que o brincar continua sendo uma atividade realizada por crianças e adolescentes em tratamento para o câncer e pode ser um recurso para o enfrentamento do processo de hospitalização. No entanto, as formas 
do brincar são limitadas, manifestando-se com maior frequência através dos jogos de tabuleiro, de adivinhação, cartas ou eletrônicos, brincadeiras de boneca, cozinha, carro, bola de gude e massinha de modelar, em detrimento a brincadeiras que envolvem movimentações corporais, como brincadeiras de correr, andar de bicicleta ou praticar esportes, as quais são limitadas por questões físicas inerentes à doença e seu tratamento e/ou pela forma como se constitui o ambiente hospitalar.

Contudo, a necessidade de brincadeiras que envolvam mais movimentos corporais, interação social e ambiental ganha destaque nos relatos dos pacientes, quando questionados sobre o que gostariam de realizar durante o período de hospitalização. Assim, o uso de recursos tecnológicos, como o videogame ativo, pode contribuir para a superação de algumas das limitações, adaptando a prática de atividades como esportes e dança, num contexto de humanização e enfrentamento da realidade hospitalar.

Nesse estudo, o tempo para a coleta de dados e a disponibilidade dos pacientes refletida no número de participantes, configuraram-se como limitações. Dessa forma, sugere-se a realização de novos estudos sobre o uso de brincadeiras que possibilitem a ampliação do movimento corporal no contexto hospitalar, visto o potencial de contribuição para o enfrentamento do período de hospitalização e a relevância das discussões sobre estes elementos na área da saúde.

\section{REFERÊNCIAS}

AJURIAGUERRA, J. Manual de psiquiatria infantil. Barcelona: Toray-Masson. Reimpressão 2005.

ALVES, D. F. S.; GUIRARDELLO, E. B.; KURASHIMA, A. Y. Estresse relacionado ao cuidado: o impacto do câncer infantil na vida dos pais. Revista Latino-Americana de Enfermagem, v.21, n.1, p. 356-362, 2013.

BARDIN, L. Análise de conteúdo. Lisboa: Edições 70; 2009.

BRASIL. Lei nº11. 104, de 21 de março de 2005. Diário Oficial, Brasília, DF, 21 mar. 2005. Disponível em: http://www.planalto.gov.br/ccivil_03/_Ato20042006/2005/Lei/L11104.htm. Acesso em: 4 fev. 2020. 
BRASIL. Lei nº13.716, de 24 de setembro de 2018. Diário Oficial, Brasília, DF, Altera a Lei $n^{\circ}$ 9.394, de 20 de dezembro de 1996 (Lei de Diretrizes e Bases da Educação Nacional), 2018. Disponível em: https://www.planalto.gov.br/ccivil_03/_ato20152018/2018/Lei/L13716.htm. Acesso em: 14 jul. 2020.

Lei n 9.394, de 20 de dezembro de 1996 (Lei de Diretrizes e Bases da Educação

Nacional). Diário Oficial, Brasília, DF, Disponível em: https://www.planalto.gov.br/ccivil_03/leis/19394.htm. Acesso em: 14 jul. 2020.

Ministério da Saúde. Programa nacional de humanização da assistência hospitalar- PNHAH. Brasília: Editora do Ministério da Saúde, 2002.

Ministério da Saúde. Secretaria de Atenção à Saúde. Núcleo Técnico da Política Nacional de Humanização. HumanizaSUS: documento base para gestores e trabalhadores do SUS. 3. ed. - Brasília: Editora do Ministério da Saúde, 2006.

BROWER, R. G. Consequences of bed rest. Critical care medicine, Mount Prospect, v. 37, n. 10, p. S422-S428, 2009.

CARVALHO, T. G. P.; FREITAS, C. M. S. M. O videogame ativo como estratégia do profissional de educação física no auxílio do tratamento de câncer infanto juvenil. Motricidade. 2018, vol. 14, SI, pp. 85-92. http://dx.doi.org/10.6063/motricidade.16243.

O olhar do paciente sobre o câncer infantojuvenil e sua percepção acerca de seus sentimentos e emoções diante do videogame ativo. Movimento, Porto Alegre., v. 24, n. 2, p. 413-426, 2018.

CATAUDELLA, D.; ZELCER, S. Psychological experiences of children with brain tumors at end of life: parental perspectives. Journal of Palliative Medicine, v. 15, n. 11, p. 1191-1197, 2012.

DIAS, R. R.; BAPTISTA, M. N.; BAPTISTA, A. S. D. Enfermaria de Pediatria: Avaliação e intervenção psicológica. In: RAAD, Alexandre José. Psicologia hospitalar: Teoria, aplicações e casos clínicos. Rio de Janeiro: Guanabara Koogan, p. p. 53-73, 2003.

DEJOURS, C. Palestra conferida na Federação dos Trabalhadores da Metalurgia, da Confederação Geral dos Trabalhadores (CGT). Revista Brasileira de Saúde Ocupacional, São Paulo, v. 14, n. 54, 1986.

DUARTE, M. L. C; ZANINI, L. N.; NEDEL, M. N. B. O cotidiano dos pais de crianças com câncer e hospitalizadas. Revista Gaúcha de Enfermagem, v.33, n.3, p. 111-118, 2012.

FLICK, U. Qualidade na pesquisa qualitativa: coleção pesquisa qualitativa. Porto Alegre: Bookman Editora, 2009.

GALVÃO, I. Henri Wallon: uma concepção dialética do desenvolvimento infantil. 23 ed. Petrópolis: Editora Vozes, 2014.

GOMES-DA-SILVA, P. N. O jogo da cultura e a cultura do jogo: por uma semiótica da corporeidade. João Pessoa: Ed. UFPB, 2011. 
Mundos sem fim do brincar- de Winnicott pra depois. In. BANDEIRA, G. (org). Viver criativo: escritos de educação com Winnicott. Curitiba: Editora CRV. 2016, p. 157-186.

GÖTTE, M. et al. Comparison of Self-Reported Physical Activity in Children and Adolescents Before and During Cancer Treatment. Pediatric blood \& cancer, Hoboken, n. 61, p.1023-1028, 2014.

HOSTERT, P.C.C.P.; ENUMO, S.R.F.; MOTTA LOSS, A. B. Brincar e problemas de comportamento de crianças com câncer de classes hospitalares. Revista Psicologia: Teoria e Prática, São Paulo-SP v.16, n.1, p.127-140, jan.- abr., 2014.

; MOTTA, A. B.; ENUMO, S. R. F. Coping da hospitalização em crianças com câncer: a importância da classe hospitalar. Estudos de Psicologia, Campinas, v.32, n.4, p. 627- 639, 2015.

ISAYAMA, H. F.; SIQUEIRA, F. T. R.; ARAÚJO, N. DE S.; PINTO, G. B.; DE SOUZA, T. R.; NUNES, L. M. O lazer na humanização hospitalar: diálogos possíveis. Licere, v. 14, n. 2, 20 jun. 2011.

LARIZZATTI, M. F. O que todo recreador precisa conhecer sobre o lazer. São Paulo: Phorte Editora, 2010.

LAZARUS, R. S.; FOLKMAN, S. Stress, appraisal and coping. New York: Springer Publishing Company, 1984.

LIMA, I. L. R. et al. Animação cultural no contexto hospitalar: relatando as vivências de acadêmicos de educação física e turismo. Revista Iberoamericana, v.2, n.1, 2008.

MALTA, J. D. S; SCHALL, V. T. Instrumento para a humanização do cuidado do câncer infanto-juvenil. Pediatria Moderna, v. 48, n.2, p.74-78, fev. 2012.

MEDINA, J. P. S.; HUNGARO, E. M.; ANJOS, R.; BRACHT, V. A Educação Física Cuida do Corpo... E "mente". Ed. 26. Papirus Editora, 2010.

MELO, L. L.; VALLE, E. R. M. A Brinquedoteca como possibilidade para desvelar o cotidiano da criança com câncer em tratamento ambulatorial. Revista da escola de enfermagem USP, São Paulo, v.44, n.2, p. 517-525, 2010.

MITRE, R. M. A; GOMES, R. A promoção do brincar no contexto da hospitalização infantil como ação de saúde. Ciência \& Saúde Coletiva, v. 9, n.1, p. 147-154, 2004.

MOTTA, A. B.; ENUMO, S. R. F. Brincar no hospital: estratégia de enfrentamento da hospitalização infantil. Psicologia em Estudo, Maringá, v. 9, n. 1, p. 19-28, 2004.

MOTA PACCIULIO SPOSITO, A. et al . O melhor da hospitalização: contribuições do brincar para o enfrentamento da quimioterapia. Av. Enferm., Bogotá, v. 36, n. 3, p. 328337, 2018.

NEHMY, R. M. Q. et al. A perspectiva dos pais sobre a obtenção do diagnóstico de leucemia linfóide aguda em crianças e adolescentes: uma experiência no Brasil. Revista Brasileira Saúde Materna Infantil. v. 11, n.3, p. 293-299, 2011. 
OLIVEIRA, R. B. G. et al. Entrevista nas pesquisas qualitativas de enfermagem pediátrica. Revista Brasileira de Enfermagem, Brasília, v.63, n.2, p. 300-306, 2010.

PAGOTTO, F. P; SILVA, R. L. "Recreação Hospitalar: Explorando o universo do trabalho voluntário na ACACCI - Vitória - ES". In: ENCONTRO NACIONAL DE RECREAÇÃO E LAZER, 18. Anais Curitiba: Confraria dos profissionais de lazer do Paraná, 2006.

PAULA, E. M. A. T. Os movimentos corporais de crianças e adolescentes nos hospitais: para além das necessidades corporais. Luminária, União da Vitória, n. 1, p. 5-16, 2013.

RAMOS, F. P.; ENUMO, S. R. F.; PAULA, K. M. P. Teoria Motivacional do Coping: Uma proposta desenvolvimentista de análise do enfrentamento do estresse. Estudos de Psicologia. Campinas, v. 32, n. 2, p. 269-279, 2015.

STENHOLM, S et al. Self-reported sleep duration and time in bed as predictors of physical func-tion decline: results from the In CHIANTI study. Sleep, Darien v. 34, n. 11, p. 1583-1593, 2011.

WINNICOTT, D. W. O brincar e a realidade. Trad. ABREU, José Octavio de Aguiar e NOBRE, Vanede. Rio de Janeiro: Imago. 1975.

WINTER, C. et al. Level of activity in children undergoing cancer treatment. Pediatric blood \& cancer, Hoboken, v. 53, n. 3, p. 438-443, 2009.

\section{Endereço dos(as) Autores(as):}

Talita Grazielle Pires de Carvalho

Escola Superior de Educação Física - Universidade de Pernambuco

R. Arnóbio Marquês, 310 - Santo Amaro

Recife - PE - 50.100-130

Endereço Eletrônico: talitagraziellepires@gmail.com

Ameliane da Conceição Reubens-Leonidio

Escola Superior de Educação Física - Universidade de Pernambuco

R. Arnóbio Marquês, 310Santo Amaro

Recife - PE - 50.100-130

Endereço Eletrônico: Ameliane.doutorado@gmail.com

Priscilla Pinto Costa da Silva

Av. Sen. Salgado Filho, 3000 - Campus Universitário

Lagoa Nova Natal - RN - 59.072-970.

Endereço Eletrônico: laprisci@gmail.com

Clara Maria Silvestre Monteiro de Freitas

Escola Superior de Educação Física - Universidade de Pernambuco

R. Arnóbio Marquês, 310 - Santo Amaro

Recife - PE - 50.100-130

Endereço Eletrônico: clarasilvestre@uol.com.br 
O Brincar Durante o Período de Hospitalização para Tratamento de Câncer Pediátrico

Talita Grazielle P. de Carvalho, Ameliane da C. Reubens-Leonidio, Priscilla Pinto C. da Silva, Clara Maria S. M. de Freitas, Pierre N. Gomes-da-Silva e Ana Raquel M. dos Santos

Pierre Normando Gomes-da-Silva

Via das Castanholas - Castelo Branco

João Pessoa - PB - 58.033-455.

Endereço Eletrônico: pierrenormandogomesdasilva@gmail.com

Ana Raquel Mendes dos Santos

Escola Superior de Educação Física - Universidade de Pernambuco

R. Arnóbio Marquês, 310 - Santo Amaro

Recife - PE - 50.100-130

Endereço Eletrônico: raquelmdss @ hotmail.com 